\title{
Retropubic, Laparoscopic, and Robot-Assisted Radical Prostatectomy: A Comparative Analysis of the Surgical Outcomes in a Single Regional Center
}

\author{
Lorenzo Giuseppe Luciani $^{\mathrm{a}} \quad$ Daniele Mattevi ${ }^{\mathrm{b}} \quad$ William Mantovanic \\ Tommaso Cai $^{\mathrm{a}}$ Stefano Chiodini ${ }^{\mathrm{a}}$ Valentino Vattovani ${ }^{\mathrm{a}}$ Marco Puglisi ${ }^{\mathrm{a}}$ \\ Daniele Tiscione $^{\mathrm{a}} \quad$ Umberto Anceschi $^{\mathrm{a}} \quad$ Gianni Malossini ${ }^{\mathrm{a}}$ \\ aDepartment of Urology, Santa Chiara Hospital, Trento; 'Urology Clinic, Integrated University Hospital, Verona; \\ 'Department of Prevention, Public Health Care Trust, Trento, Italy
}

\section{Key Words}

Open retropubic prostatectomy • Prostate cancer •

Robotic-assisted laparoscopic prostatectomy $\cdot$ Complication

\begin{abstract}
Background: To compare the surgical outcomes of radical prostatectomy (RP) performed via 3 different approaches: retropubic (RRP), laparoscopic-assisted (LRP), and robot-assisted (RARP), in a single non-academic regional center by a single surgeon. Materials and Methods: The data of patients undergoing RP from 2005 to 2014 were reviewed. The standard approach changed through the years: $\operatorname{RRP}(n=380$, years 2005 to 2008), LRP ( $n=240$, years 2009 to 2011), and RARP ( $n=262$, years 2012 to 2014). Our analysis included the last consecutive $100 \mathrm{RP}$ for each surgical technique by a single surgeon. A logistic regression model adjusted for preand postoperative variables was done to evaluate whether transfusion, conversion, and post-operative complication rates were influenced by the approach. Results: RARP was associated with significantly lower blood loss (400 vs. 600 and $600 \mathrm{ml}$, respectively), transfusion ( 6 vs. 21 and 21\%, respectively), and shorter hospital stay ( 6 vs. 7 and 8 days, respectively), compared to LRP and RRP, and a lower conversion rate ( 1 vs. $12 \%$ ) compared to LRP. Multivariate analysis adjusted for confounders confirmed that the risk of transfusion and conversion was significantly lower in the RARP group compared to the LRP and RRP groups. The RARP group
\end{abstract}

\section{KARGER}

Fax +4161306 1234

E-Mail karger@karger.com

www.karger.com
(C) 2017 S. Karger AG, Basel

Accessible online at: www.karger.com/cur was also associated with a significantly lower risk of complications compared to the RRP group and with a trend in favor of the RARP group compared to the LRP group. The 1-year continence rate was significantly higher in the RARP group compared to the RRP and LRP groups ( 80 vs. 72 and $68 \%$, respectively). Conclusion: The surgical approach affected the operative outcomes in a regional setting. The advantages of RARP over RRP (complications, transfusion, conversion, hospital stay, 1-year continence) were over LRP as well, with the only exception being complications.

Copyright $\odot 2017$ S. Karger AG, Basel

\section{Introduction}

Radical prostatectomy (RP) is the standard treatment for patients with clinically localized prostate cancer (PCa) (cT1-T2) and a life expectancy of $>10$ years [1]. Different approaches to this procedure have been proposed: retropubic (RRP), laparoscopic (LRP), and robot-assisted radical prostatectomy (RARP).

Whereas open RRP has been considered the reference standard for surgical treatment [2], minimally invasive procedure have been introduced with the intention of minimizing peri- and post-operative morbidities [3]. LRP was proposed by Schuessler et al. [4] in 1992 and it was standardized by Gaston in 1997 [5]. Several centers in Europe developed LRP but with no real worldwide diffusion, being a technically demanding procedure [6].
Lorenzo G. Luciani
Daniele Mattevi

Urology Clinic

Integrated University Hospital

P.le Stefani 1

IT-37126, Verona (Italy)

E-Mail danielemattevi85@gmail.com 
Table 1. Preoperative, operative, and pathological data.

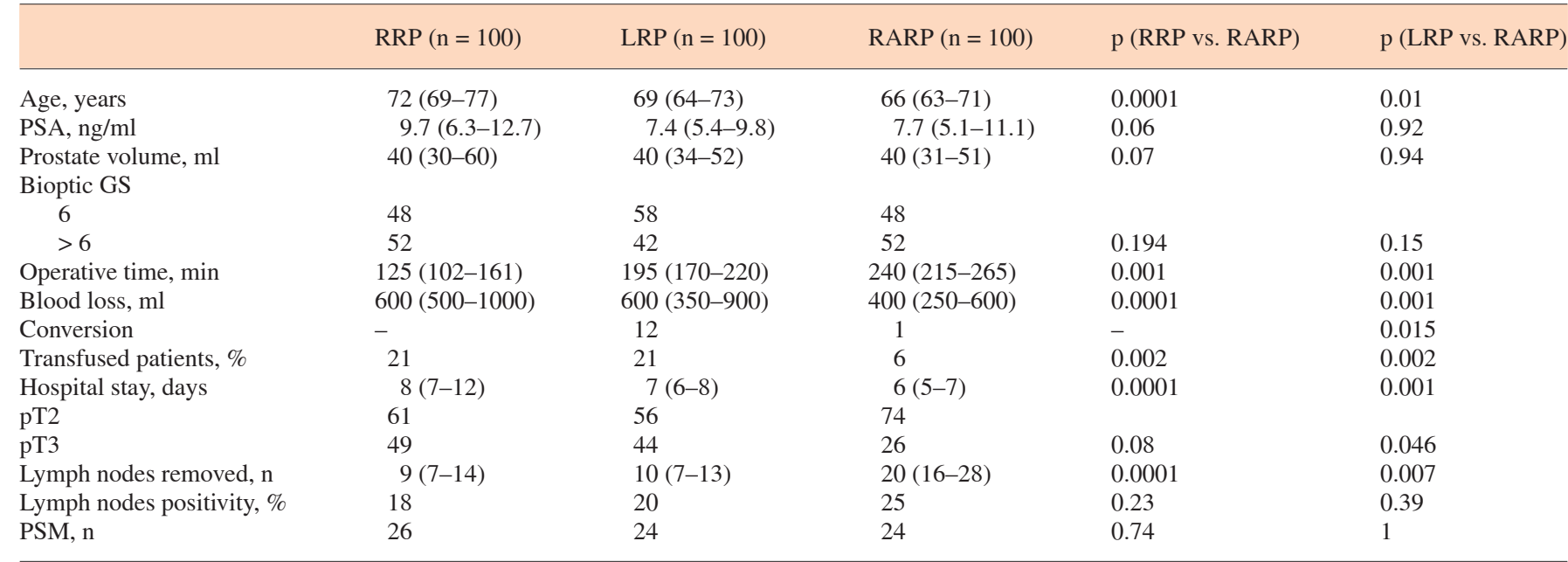

Values are expressed as the median and interquartile ranges. GS = Gleason score; PSM = positive surgical margins.

In May 2000 Binder et al. [7] reported the first LRP assisted by a robotic master-slave system (da Vinci, Intuitive Surgical, Sunnyvale, CA, USA). The feasibility, safety, and early functional efficacy of RARP have been well documented. A recent population-based analysis comparing RARP and RRP procedures undertaken between 2003 and 2005 concluded that men undergoing RARP experienced significantly fewer 30-day complications, blood transfusions, and shorter lengths of stay [8]. Most comparative studies considered RRP versus RARP or versus LRP. Moreover, these studies rarely used a formal reporting system for complications and the procedures were performed by multiple surgeons [9].

The aim of this study was to compare the complication rate and perioperative data of 3 different approaches (RRP, LRP, and RARP) after the initial learning curve in a regional hospital by a single surgeon.

\section{Materials and Methods}

\section{Study Design and Data Collection}

We performed a retrospective analysis from a prospectively maintained database collecting data on 902 patients surgically treated for PCa over 10 years (from January 2005 to December 2014).

The standard surgical approach changed through the years: RRP ( $\mathrm{n}=380$, years 2005-2008), LRP $(\mathrm{n}=240$, years 20092011), and RARP ( $n=262$, years 2012-2014). Our analysis included the last consecutive $100 \mathrm{RP}$ for each surgical technique by a single surgeon.

Retropubic, Laparoscopic, and

Robot-Assisted Radical Prostatectomy:

An Analysis of Surgical Outcomes
The preoperative workup for the all groups consisted of serum prostate-specific antigen (PSA) measurement, a minimum of 12core biopsies for cancer detection, and staging accordingly to the EAU guidelines [1]. Prostate volume and clinical stage were evaluated by transrectal ultrasonography and digital rectal examination, respectively. Tumors were graded histologically according to the Gleason grading system [10], and the pathological stage was defined according to the TNM staging classification [11]. No patient had undergone preoperative radiotherapy or neoadjuvant androgen deprivation therapy. Our study was over a long time span (10 years): patients were selected for a nerve-sparing approach on an individual basis according to the current EAU guidelines. Since 2011 an extended lymph node dissection was performed according to Briganti's nomogram [12]. A limited lymphadenectomy was performed in intermediate and high risk patients according to the D'Amico classification from year 2005 to 2010. We evaluated the following perioperative variables: operative time, estimated blood loss (EBL), transfusion rate, intra- and peri-operative (within the first 30 days) complications, and hospital stay. Medical and surgical complications were collected for all patients through a combination of institutional electronic medical records and operative notes. Complications were classified according to the Clavien-Dindo system [13]. Patients were usually transfused in case of hemoglobin level $<8 \mathrm{~g} / \mathrm{dl}$ in healthy patients and < $9 \mathrm{~g} / \mathrm{dl}$ in patients with ischemic cardiac disease. The $12-\mathrm{month}$ continence rate, defined as the use of no or one safety pad, was evaluated.

\section{Surgical Details}

$R R P$ All operations were performed extraperitoneally, with a small umbilico-pubic incision (minilaparotomy) [14]. The procedure was performed according to the technique described by Walsh [15]. The vesico-urethral anastomosis was made via an interrupted suture of 5 stitches. 
LRP An extraperitoneal 5-trocar approach was used. The vesico-urethral anastomosis was made via 2 running sutures with 2-0 Monocryl according to the technique described by Van Velthoven [16].

RARP The doors of the 4-arm robot (da Vinci Intuitive Surgical Inc, Sunnyvale, CA, USA) were placed in a pattern like a fan. All cases were performed by transperitoneal access. The lateral pedicles were controlled using Hem-o-lock clips and bipolar forceps as little as possible and as far away as possible from the neurovascular bundles. The vesico-urethral anastomosis was made with 2 running sutures with 3-0 V-Loc.

\section{Statistical Analysis}

Distribution of continuous variables are reported as the median and interquartile range $\left(25^{\text {th }} ; 75^{\text {th }}\right.$ percentiles). Categorical variables are presented as numbers and percentages. The comparison between subgroups (surgical approaches) was done using the Student $t$ test or Mann-Whitney U test for continuous variables. Qualitative data were compared by the $\chi^{2}$ test or Spearman correlation.

Univariate and multivariate analysis was done by the logistic regression model to evaluate significant predictors of transfusion, conversion, and postoperative complication rates, and odds ratios (OR) and the $95 \%$ confidence interval (CI) were calculated. Two different regression models were calculated and adjusted for preoperative (age, PSA, prostate volume, and bioptic Gleason score) and postoperative data (pT stage and pathological Gleason score) plus age and PSA. P values were considered significant when less than or equal to 0.05 . All tests were two-sided. All analyses were done using the SPSS software (IBM Corp., Armonk, N.Y.).

\section{Results}

Preoperative Characteristics and Pathological Data

The preoperative characteristics of the 3 groups are shown in table 1. Patients undergoing RARP were significantly younger compared to those undergoing LRP and RRP, whereas PSA and prostate volume were comparable in the 3 groups. The bioptic Gleason score was also comparable in the 3 groups.

\section{Operative and Perioperative Results}

Operative and perioperative data are shown in table 1. The median operative time was significantly shorter in the RRP group compared to the LRP and RARP groups (125 vs. 195 and 240 min, respectively). The median EBL was significantly lower in the RARP group compared to the RRP and LRP groups (400 vs. 600 and $600 \mathrm{ml}$, respectively) as well as the transfusion rate (6 vs. 21 and $21 \%$, respectively). The conversion rate was statistically higher in the LRP group compared to the RARP group (12 vs. 1\%). The hospital stay was statistically shorter in the RARP group compared to the other 2 surgical techniques. The 1-year continence rate was 72,68 , and $80 \%$ after RRP, LRP, and RARP, respectively.
Table 2. Complications graded according to the Clavien-Dindo classification and treatments

\begin{tabular}{|c|c|c|c|}
\hline & RRP & LRP & RARP \\
\hline I & 13 & 11 & 14 \\
\hline II & 27 & 24 & 13 \\
\hline Blood loss (transfusion) & 21 & 21 & 6 \\
\hline Hypertension (antihypertensive drugs) & 1 & - & 3 \\
\hline Atrial fibrillation (antiarrhytmics) & 2 & 2 & - \\
\hline Pneumonia (antibiotics) & 2 & 1 & - \\
\hline Respiratory failure (oxygen) & 2 & - & - \\
\hline Fever (antibiotics) & 1 & - & 3 \\
\hline IIIa & 1 & 3 & - \\
\hline Acute urinary retention (catheterization) & 1 & 2 & - \\
\hline Acute urinary retention (urethral calibration) & - & 1 & - \\
\hline IIIb & 6 & 3 & 1 \\
\hline Hydronephrosis (stent) & 1 & 1 & 1 \\
\hline Pelvic hematoma (reintervention) & 3 & 1 & - \\
\hline Ureteral injury (ureter repair) & 1 & 1 & - \\
\hline Anastomosis dehiscence (reintervention) & 1 & - & - \\
\hline IV & - & - & - \\
\hline $\mathrm{V}$ & - & - & - \\
\hline Total & 47 & 41 & 28 \\
\hline
\end{tabular}

\section{Pathological Data}

Patients undergoing RARP had a lower rate of pathologically locally advanced disease compared to LRP and RRP. The overall positive surgical margins rate was 26, 24 , and $24 \%$ after RRP, LRP and RARP, respectively. The median number and positivity percentage of the lymph nodes removed was $9(18 \%)$ in the RRP, $10(20 \%)$ in LRP, and 20 (24\%) in RARP groups.

\section{Complications}

Complications according to the Clavien-Dindo classification are summarized in table 2, including the type of complications and their management. Grade I complications were similar in the 3 groups and included pain and transient fever. Grade II complications were statistically lower in the RARP group compared to the RRP and LRP groups (13 vs. 27 and 24\%, respectively). The rate of Grade III complications was also lower in the RARP group $(\mathrm{n}=1)$ compared to the RRP $(\mathrm{n}=7)$ and LRP groups $(n=6)$. No Grade IV or V complications were reported.

\section{Comparative Results Adjusted for Patients \\ Characteristics}

The results of the univariate and multivariate analysis adjusted for pre- and post-operative variables (age, PSA, prostatic volume, pathological stage, and pre- and post- 
Table 3. Univariate and multivariate analysis using a logistic regression model to evaluate the risk of transfusion, overall complications, and conversion

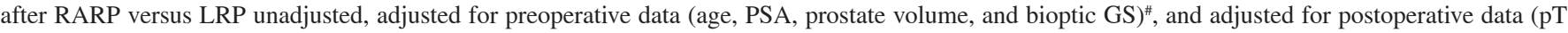
stage and pathological GS) plus age and PSA*

\begin{tabular}{|c|c|c|c|c|c|c|}
\hline & \multicolumn{2}{|c|}{ RARP vs. LRP unadjusted } & \multicolumn{2}{|c|}{ RARP vs. LRP adjusted" } & \multicolumn{2}{|c|}{ RARP vs. LRP adjusted* } \\
\hline & $\overline{\mathrm{OR}}(95 \% \mathrm{CI})$ & $\mathrm{p}$ & OR $(95 \% \mathrm{CI})$ & $\mathrm{p}$ & OR $(95 \% \mathrm{CI})$ & $\mathrm{p}$ \\
\hline Transfusion & $0.24(0.09-0.62)$ & 0.003 & $0.29(0.11-0.80)$ & 0.016 & $0.30(0.11-0.81)$ & 0.016 \\
\hline Overall complications & $0.54(0.32-1.05)$ & 0.074 & $0.58(0.30-1.11)$ & 0.097 & $0.69(0.37-1.29)$ & 0.097 \\
\hline Conversion & $0.33(0.14-0.78)$ & 0.012 & $0.34(0.13-0.87)$ & 0.024 & $0.41(0.16-1)$ & 0.024 \\
\hline
\end{tabular}

Table 4. Univariate and multivariate analysis using a logistic regression model to evaluate the risk of transfusion and overall complications after RARP versus RRP unadjusted, adjusted for preoperative data (age, PSA, prostate volume, and bioptic Gleason score) ${ }^{\#}$, and adjusted for postoperative data (pT stage and pathological Gleason score) plus age and PSA"

\begin{tabular}{|c|c|c|c|c|c|c|}
\hline & \multicolumn{2}{|c|}{ RARP vs. RRP unadjusted } & \multicolumn{2}{|c|}{ RARP vs. RRP adjusted ${ }^{\#}$} & \multicolumn{2}{|c|}{ RARP vs. RRP adjusted* } \\
\hline & $\overline{\mathrm{OR}}(95 \% \mathrm{CI})$ & $\mathrm{p}$ & OR $(95 \% \mathrm{CI})$ & $\mathrm{p}$ & $\overline{\mathrm{OR}}(95 \% \mathrm{CI})$ & $\mathrm{p}$ \\
\hline Transfusion & $0.24(0.09-0.62)$ & 0.003 & $0.33(0.10-1.09)$ & 0.069 & $0.32(0.11-0.95)$ & 0.041 \\
\hline Overall complications & $0.44(0.24-0.79)$ & 0.006 & $0.38(0.17-0.85)$ & 0.018 & $0.45(0.22-0.92)$ & 0.028 \\
\hline
\end{tabular}

operative Gleason score) are shown in table 3 (RARP vs. LRP) and table 4 (RARP vs. RRP). The risk of transfusion and conversion was significantly lower in the RARP group compared to the LRP group and RRP groups. RARP was also associated with a significantly lower risk of complications compared to RRP and with a trend in favor of RARP as compared to LRP.

\section{Discussion}

RP is the treatment of choice with curative intent in $\mathrm{PCa}$ and RRP is accepted as the reference [1]. After the standardization of the LRP technique [6], the use of minimally invasive procedures has gradually risen. However, because of the steep learning curve of LRP, few centers succeeded to improve a structured program with high caseloads, and RARP is displacing all other surgical approaches [17]. The evolution of the surgical approach to $\mathrm{RP}$ is well reflected at our department, where the changeover to RARP occurred after LRP was the standard of care for 3 years (2009-2011). From the beginning of 2012, pure LRP has been supplanted by the robotic approach.

Several studies compared the surgical outcomes of the 3 approaches to RP, mainly as a one-to-one comparison of RRP versus LRP or RRP versus RARP. Only 3 studies reported the results of the 3 approaches in sin-

Retropubic, Laparoscopic, and

Robot-Assisted Radical Prostatectomy:

An Analysis of Surgical Outcomes gle academic and non-academic institutions reporting a relatively low $(\mathrm{n}=86, \mathrm{n}=239)[18,19]$ or non-homogeneously distributed number of patients among the 3 techniques [20], and only the series reported by Akand was performed by a single surgeon.

Our study compared the outcomes of RP performed by a single surgeon in a regional hospital, including a series of consecutive patients selected after completing the learning curve of each technique. A logistic regression model was used in order to control the differences in baseline demographic and pathological data among cohorts.

Recent data from the US showed a migration from high to low volume centers combined with a higher complication rate in low-volume centers [21]. From this point of view, it becomes helpful to compare the results from a regional setting with the results from larger centers.

Intraoperative bleeding has been noticeably reduced by clear visualization and better appreciation of the anatomy of the dorsal venous complex, apex, and neurovascular bundles allowed by a robotic system $[9,22]$. In our study, the median EBL was significantly lower in the RARP group compared to the RRP and LRP groups (400 vs. 600 and $600 \mathrm{ml}$, respectively), as well as the rate of transfusion (6 vs. 21 and $21 \%$, respectively), confirming previous results from comparative studies among the 3 approaches [18-20] as well as from one-to-one comparisons [9]. 
Another parameter supporting the benefit of the intuitive nature of the robotic technique is the rate of conversion to open surgery. In fact, although relatively uncommon, life-threatening bleeding might require an emergency conversion to an open procedure. Most studies report conversion rates of $2-8 \%$ for LRP and $0-1 \%$ for RARP, which is consistent with our findings (12 vs. $1 \%)$ [23].

The analysis of our pathological data showed no difference in positive surgical margins among the 3 techniques. A greater number of lymph nodes were removed with the robotic approach, confirming previous studies [24], whereas lymph node positivity was comparable among the 3 groups, with a trend in favor of RARP.

As far as complications are concerned, grade I complications according to Clavien-Dindo were similar in the 3 groups, whereas Grade II complications were lower in the RARP group compared to the RRP and LRP groups (13 vs. 27 and 24\%, respectively). Similar results were reported for Grade III complications (1 vs. 6 and 3\%, respectively). No patient undergoing RP experienced Grade IV or V complications. The overall rate of complications was significantly lower in the RARP group compared to the RRP group $(\mathrm{p}=0.005)$ and with a trend in favor of RARP over LRP as well, confirming previous results [25].

In general, the hospital stay was higher than that reported in the literature [9], reflecting different management pathways. Specifically, our patients stay in hospital until the urinary catheter is removed, whereas patients in the United States are discharged earlier [9]. In our study, the hospital stay was significantly lower in the RARP group compared to the other surgical techniques, resulting in greater patient's satisfaction and lower costs. The reduced tissue trauma related to RARP, as indicated by the more limited increase in acute-phase inflammatory mediators interleukin- 6 and $C$ reactive protein [26] and serum cortisol levels [27] associated with this approach might imply an improvement in several perioperative parameters, eventually affecting hospital stay.

Moreover, multivariate analysis revealed that risk of transfusion, conversion, and overall complications was not influenced by pre- and post-operative variables. The only exception was the overall complications of LRP versus RARP, where a distinct trend in favor of the RARP technique occurred (table 3 ). To our knowledge, this is the only study comparing the 3 techniques using a linear regression model in order to control the biases from preand post-operative variables. However, operative time was significantly longer in the RARP group as compared to the other 2 techniques, duplicating similar results from the literature (160-330 minutes for RARP) [25]. In addition, our data confirmed the operative results from a recent single center randomized study between RRP and RARP performed by 2 different surgeons [28].

Our study has some limitations. First, this is a non-randomized retrospective study comparing different surgical approaches. However, many patients are unwilling to be randomly assigned to a particular surgical treatment and are usually attracted by the most modern surgical procedure or choose a procedure based on personal preferences for a specific surgeon. Second, our study involved a relatively low number of patients with inherent biases related to data collection. In this case, selection biases were partially limited by the inclusion criteria: the last consecutive $100 \mathrm{RP}$ for each surgical technique were included. Also, indications for surgery, operative techniques, perioperative management, and data collection, should be relatively homogeneous in a single center. Consequently, the present study provides a realistic view of daily clinical practice.

\section{Conclusion}

The introduction of RARP at a single non-academic regional center improved the rate of surgical complications, blood transfusion, and conversion as well as hospital stay, as compared to RRP and LRP. However, a significantly longer operative time was noted. These advantages were confirmed at multivariate analysis adjusted for pre- and post-operative variables, with the exception of the overall rate of complications, where the favorable trend of RARP over LRP did not reach statistical significance. Our results show the superiority of RARP over RRP and LRP with respect to surgical outcomes, which persisted after adjusting for confounders. Moreover, the 1 -year continence rate was significantly higher in the RARP group. Further controlled studies with a higher caseload and long oncological follow-up are needed to designate RARP as the standard of care. 


\section{References}

1 Heidenreich A, Bastian PJ, Bellmunt J, Bolla M, Joniau S, van der Kwast T, Mason M, Matveev V, Wiegel T, Zattoni F, Mottet N: EAU guidelines on prostate cancer. part 1: screening, diagnosis, and local treatment with curative intent-update 2013. Eur Urol 2014;65:124-137.

2 Finkelstein J, Eckersberger E, Sadri H, Taneja SS, Lepor H, Djavan B: Open versus laparoscopic versus robot-assisted laparoscopic prostatectomy: the European and US experience. Rev Urol 2010;12:35-43.

3 Duffey B, Varda B, Konety B: Quality of evidence to compare outcomes of open and robot-assisted laparoscopic prostatectomy. Curr Urol Rep 2011;12:229-236.

4 Schuessler WW, Schulam PG, Clayman RV, Kavoussi LR: Laparoscopic radical prostatectomy: initial short-term experience. Urology 1997;50:854-857.

5 Curto F, Benijts J, Pansadoro A, Barmoshe S, Hoepffner JL, Mugnier C, Piechaud T, Gaston R: Nerve sparing laparoscopic radical prostatectomy: our technique. Eur Urol 2006;49:344-352.

6 Guillonneau B, Cathelineau X, Barret E, Rozet F, Vallancien G: Laparoscopic radical prostatectomy. Preliminary evaluation after 28 interventions. Presse Med 1998;27:15701574.

7 Binder J, Kramer W: Robotically-assisted laparoscopic radical prostatectomy. BJU Int 2001;87:408-410.

8 Hu JC, Hevelone ND, Ferreira MD, Lipsitz SR, Choueiri TK, Sanda MG, Earle CC: Patterns of care for radical prostatectomy in the United States from 2003 to 2005. J Urol 2008;180:1969-1974.

9 Ficarra V, Novara G, Artibani W, Cestari A, Galfano A, Graefen M, Guazzoni G, Guillonneau B, Menon M, Montorsi F, Patel V, Rassweiler J, Van Poppel H: Retropubic, laparoscopic, and robot-assisted radical prostatectomy: a systematic review and cumulative analysis of comparative studies. Eur Urol 2009;55:1037-1063.

10 Epstein JI, Amin M, Boccon-Gibod L, Egevad L, Humphrey PA, Mikuz G, Newling D, Nilsson S, Sakr W, Srigley JR, Wheeler TM, Montironi R: Prognostic factors and reporting of prostate carcinoma in radical prostatectomy and pelvic lymphadenectomy specimens. Scand J Urol Nephrol Suppl 2005; 216:34-63.

11 Edge SB, Compton CC: The American Joint Committee on Cancer: the 7th edition of the AJCC cancer staging manual and the future of TNM. Ann Surg Oncol 2010;17:14711474.
12 Briganti A, Larcher A, Abdollah F, Capitanio U, Gallina A, Suardi N, Bianchi M, Sun M, Freschi M, Salonia A, Karakiewicz PI, Rigatti P, Montorsi F: Updated nomogram predicting lymph node invasion in patients with prostate cancer undergoing extended pelvic lymph node dissection: the essential importance of percentage of positive cores. Eur Urol 2012;61:480-487.

13 Dindo D, Demartines N, Clavien PA: Classification of surgical complications: a new proposal with evaluation in a cohort of 6336 patients and results of a survey. Ann Surg 2004;240:205-213.

14 Marshall FF, Chan D, Partin AW, Gurganus R, Hortopan SC: Minilaparotomy radical retropubic prostatectomy: technique and results. J Urol 1998;160:2440-2445.

15 Walsh PC: Anatomic radical prostatectomy: evolution of the surgical technique. J Urol 1998;160:2418-2424.

16 Van Velthoven RF, Ahlering TE, Skarecky DW, Clayman RV: Technique for laparoscopic running urethrovesical anastomosis: the single knot method. Urology 2003;61: 699-702.

17 Tyson M, Scottsdale AZ, Andrews P: Radical Prostatectomy Trends in the United States: 1998-2011. Abstract MP4-11. Annual Meeting of the American Urological Association (AUA), New Orleans, May 15, 2015.

18 Durand X, Vaessen C, Bitker MO, Richard F: Retropubic, laparoscopic and robot-assisted total prostatectomies: comparison of postoperative course and histological and functional results based on a series of 86 prostatectomies. Prog Urol 2008;18:60-67.

19 Drouin SJ, Vaessen C, Hupertan V, Comperat E, Misraï V, Haertig A, Bitker MO, Chartier-Kastler E, Richard F, Rouprêt M: Comparison of mid-term carcinologic control obtained after open, laparoscopic, and robot-assisted radical prostatectomy for localized prostate cancer. World J Urol 2009; 27:599-605.

20 Akand M, Celik O, Avci E, Duman I, Erdogru T: Open, laparoscopic and robot-assisted laparoscopic radical prostatectomy: comparative analysis of operative and pathologic outcomes for three techniques with a single surgeon's experience. Eur Rev Med Pharmacol Sci 2015;19:525-531.
21 Sammon J, Abdollah F, Klett D: Diminishing returns of robotic diffusion: complications following robot-assisted radical prostatectomy. Abstract PD6-12. Annual Meeting of the American Urological Association (AUA), New Orleans, May 15, 2015.

22 Stav K, Rahimi-Levene N, Lindner A, Siegel YI, Zisman A: Retropubic radical prostatectomy: associated blood loss and transfusion requirements--a two-decade perspective review. Isr Med Assoc J 2005;7:103-106.

23 Ahlering TE: Robotic versus laparoscopic radical prostatectomy. Nat Clin Pract Urol 2004;1:58-59.

24 Tang K, Jiang K, Chen H, Chen Z, Xu H, Ye Z: Robotic vs. retropubic radical prostatectomy in prostate cancer: a systematic review and an meta-analysis update. Oncotarget 2016;10.

25 De Carlo F, Celestino F, Verri C, Masedu F, Liberati E, Di Stasi SM: Retropubic, laparoscopic, and robot-assisted radical prostatectomy: surgical, oncological, and functional outcomes: a systematic review. Urol Int 2014;93:373-383.

26 Fracalanza S, Ficarra V, Cavalleri S, Galfano A, Novara G, Mangano A, Plebani M, Artibani W: Is robotically assisted laparoscopic radical prostatectomy less invasive than retropubic radical prostatectomy? Results from a prospective, unrandomized, comparative study. BJU Int 2008;101:1145-1149.

27 Porcaro AB, Molinari A, Terrin A, De Luyk $\mathrm{N}$, Baldassarre R, Brunelli M, Cavalleri S, Cerruto MA, Gelati M, Salvagno GL, Guidi GC, Artibani W: Robotic-assisted radical prostatectomy is less stressful than the open approach: results of a contemporary prospective study evaluating pathophysiology of cortisol stress-related kinetics in prostate cancer surgery. J Robot Surg 2015;9:249-255.

28 Yaxley JW, Coughlin GD, Chambers SK, Occhipinti S, Samaratunga H, Zajdlewicz L, Dunglison N, Carter R, Williams S, Payton DJ, Perry-Keene J, Lavin MF, Gardiner RA: Robot-assisted laparoscopic prostatectomy versus open radical retropubic prostatectomy: early outcomes from a randomised controlled phase 3 study. Lancet 2016;388:1057-1066. 